\title{
An X-ray characterization of the central region of the supernova remnant G332.5-5.6
}

\author{
A. E. Suárez ${ }^{1,2}$, J.A. Combi ${ }^{1,2}$, J.F. Albacete-Colombo ${ }^{3}$, S. Paron ${ }^{4}$, F. García ${ }^{1,2}$, and M. Miceli ${ }^{5}$ \\ ${ }^{1}$ Instituto Argentino de Radioastronomía (CCT La Plata, CONICET), C.C.5, (1894) Villa Elisa, Buenos Aires, Argentina \\ e-mail: aesuarez@iar-conicet.gov.ar \\ ${ }^{2}$ Facultad de Ciencias Astronómicas y Geofísicas, Universidad Nacional de La Plata, Paseo del Bosque, B1900FWA La Plata, \\ Argentina \\ e-mail: jcombi@fcaglp.unlp.edu.ar \\ 3 Dto de Investigación en Ciencias Exactas, Naturales e Ingeniería. Universidad Nacional de Rio Negro, \\ Don Bosco y Leloir (CP 8500) Viedma, Argentina \\ e-mail: albacete.facundo@conicet.gov.ar \\ ${ }^{4}$ Instituto de Astronomía y Física del Espacio (IAFE), CC 67, Suc. 28, 1428 Buenos Aires, Argentina \\ 5 INAF-Observatorio Astronomico di Palermo, P.za del Parlamento 1, 90134 Palermo, Italy
}

Received 8 June 2015 / Accepted 1 September 2015

\begin{abstract}
Aims. We present an X-ray analysis of the central region of supernova remnant (SNR) G332.5-5.6 through an exhaustive analysis of $X M M$-Netwon observations with complementary infrared observations. We characterize and discuss the origin of the observed X-ray morphology, which presents a peculiar plane edge over the west side of the central region.

Methods. The morphology and spectral properties of the X-ray SNR were studied using a single full frame XMM-Newton observation in the 0.3 to $10.0 \mathrm{keV}$ energy band. Archival infrared WISE observations at 8,12 and $24 \mu \mathrm{m}$ were also used to investigate the properties of the source and its surroundings at different wavelengths.

Results. The results show that the extended X-ray emission is predominantly soft $(0.3-1.2 \mathrm{keV})$ and peaks around $0.5 \mathrm{keV}$, which shows that it is an extremely soft SNR. X-ray emission correlates very well with central regions of bright radio emission. On the west side the radio/X-ray emission displays a plane-like feature with a terminal wall where strong infrared emission is detected. Our spatially resolved X-ray spectral analysis confirms that the emission is dominated by weak atomic emission lines of $\mathrm{N}, \mathrm{O}, \mathrm{Ne}$, and $\mathrm{Fe}$, all of them undetected in previous X-ray studies. These characteristics suggest that the X-ray emission is originated in an optically thin thermal plasma, whose radiation is well fitted by a non-equilibrium ionization collisional plasma (VNEI) X-ray emission model. Our study favors a scenario where G332.5-5.6 is expanding in a medium with an abrupt density change (the wall), likely a dense infrared emitting region of dust on the western side of the source.
\end{abstract}

Key words. ISM: individual objects: G332.5-5.6 - ISM: supernova remnants - X-rays: ISM - radiation mechanisms: thermal

\section{Introduction}

The energy released by supernovae (SNs) events is of great importance for our understanding of the physics and chemistry of the interstellar medium (ISM). When supernova remnants (SNRs) interact with ambient gas and dust, shocks compress, heat up, ionize, and dissociate the molecular medium. Depending upon the composition of the surrounding ISM, the SNRs can display a vast range of shapes (e.g., Whiteoak \& Green 1996).

The southern SNR G332.5-5.6, which exhibits an unusual trident radio appearance (Reynoso et al. 2007; Stupar et al. 2007), is particularly interesting. This SNR lies at intermediate galactic latitude $(l, b)=\left(332.5^{\circ},-5.6^{\circ}\right)$; its radio emission is composed of two, almost parallel, outer ridges, and a peculiar central region, which is oriented in the NW-SE direction and presents strong correlation with X-ray emission observed by ROSAT in the soft energy range, between 0.1 and $1.2 \mathrm{keV}$ (Reynoso et al. 2007). Because of its spectral characteristics, SNR G332.5-5.6 has been classified as an unusual subclass of thermal composite SNRs.
Reynoso et al. (2007) studied this remnant using radio observations at different frequencies $(1384 \mathrm{MHz}, 1704 \mathrm{MHz}$, $2496 \mathrm{MHz}$, and $2368 \mathrm{MHz}$ ) obtained with the Australian Telescope Compact Array (ATCA) and HI $\lambda 21 \mathrm{~cm}$ line observations from the Southern Galactic Plane Survey (McClure-Griffiths et al. 2009). They analyzed the radio morphology, polarization, and the distribution of the radio spectral index of the emission with these data. As a result, Reynoso et al. found that the source presents an average fractional polarization of $\sim 35$ percent, and a spectral index $\alpha=-0.7 \pm 0.2$. In addition, they were able to estimate a distance of $3.4 \mathrm{kpc}$, which implies a linear size of $\sim 30 \mathrm{pc}$ and a height over the plane of $330 \mathrm{pc}$, using $\mathrm{HI} \lambda 21 \mathrm{~cm}$ line in absorption against the radio continuum emission. Throughout this paper, a mean distance of $3.4 \mathrm{kpc}$ is assumed.

At the same time, Stupar et al. (2007), carried out radio and optical studies of the SNR and background sources, using multiple optical observations from the South African Astronomical Observatory (SAAO), observations from AAO/UKST $\mathrm{H} \alpha$ survey (Parker et al. 2005), and radio data at $843 \mathrm{MHz}$ (Bock et al. 1999), $1384 \mathrm{MHz}, 2368 \mathrm{MHz}$, and $4850 \mathrm{MHz}$ 
(Griffith et al. 1993). Their results confirmed the nonthermal nature of the source and the presence of shock-heated gas through strong SII lines relative to $\mathrm{H} \alpha$, and provided strong correlation between $\mathrm{H} \alpha$ and radio emission. A very recent paper by Zhu et al. (2015) presents a study of the X-ray properties of a fraction of the central region of SNR G332.5-5.6 using Suzaku data, which is not affected by straylight effect in that instrument.

With the unprecedented capabilities of the XMM-Newton telescope, it is now possible to perform high-quality imaging and spatially resolved spectroscopy, which are particularly well suited to the study of SNRs, even more well suited for unusual X-ray morphology SNRs, such as G332.5-5.6. In this paper, we present the results of the analysis of X-ray data taken with XMM-Newton, as well as a multiwavelength analysis made with the use of observations available from Two Micron All Sky Survey (2MASS; Skrutskie et al. 2006) and the Wide-field Infrared Survey Explorer (WISE; Wright et al. 2010).

The structure of the paper is as follows: in Sect. 2, we describe the $X M M$-Newton observations and the data reduction process. In Sect. 3, we present the results of our X-ray data analysis, including X-ray images, spectra, mean photon energy map, and the study of the IR data obtained with WISE. In Sect. 4, we discuss a possible scenario to explain the origin of the observed central morphology, and finally, we summarize our main conclusions in Sect. 5. There is additional information on Fermi data analysis in the appendix.

\section{X-ray observations and data reduction}

The SNR G332.5-5.6 has been observed by XMM-Newton satellite with the European Photon Imaging Camera (EPIC). This detector has two cameras, namely MOS1, MOS2 (Turner et al. 2001) and one PN camera (Struder et al. 2001), all of which operate in full frame mode in the $0.2-15 \mathrm{keV}$ energy range. The observation, Obs-Id 0603220201, was centered at $\left(\alpha_{\mathrm{J} 2000.0}=\right.$ $\left.16 \mathrm{~h} 42 \mathrm{~m} 55 \mathrm{~s}, \delta_{\mathrm{J} 2000.0}=-54^{\circ} 31^{\prime} 00^{\prime \prime}\right)$. It was taken with medium filter, Prime Full Window observation mode, and offset on-axis.

The data were analyzed with the XMM-Newton Science Analysis System (SAS) version 13.0.0 and the latest calibrations files. The raw data were processed with EMPROC and EPPROC tasks to obtain a filtered list of events. We searched for high background periods, which affect 4.9, 3.6, and $6 \mathrm{ks}$ of the observation, for the MOS1, MOS2, and PN cameras, respectively. Thus, the net exposure time of the observation was slightly reduced to $33.8,35.2$, and $27.1 \mathrm{ksec}$. To create images, spectra, and light curves, we selected events with FLAG $=0$, and PATTERNS $\leq 12$ and 4 for MOS and PN cameras, respectively.

\section{Results}

\subsection{X-ray images}

The SNR emits in the 0.3 to $1.2 \mathrm{keV}$ soft X-ray band. X-ray images were obtained in three different energy bands: soft $(0.3-0.5 \mathrm{keV})$, medium $(0.5-0.8 \mathrm{keV})$, and hard $(0.8-1.2 \mathrm{keV})$. We limited the analysis of XMM-Newton to MOS2 and pn cameras only. Figure 1 shows an X-ray image of G332.5-5.6 taken from MOS2 camera in the soft energy range $(0.3-1.0 \mathrm{keV})$. Data from MOS2 camera were exposure-vignetting corrected, and smoothed with a Gaussian kernel for a bin size of 3 pixels.

The image reveals that the X-ray morphology of G332.5-5.6 is consistent with central extended emission as seen by Reynoso et al. (2007) and Stupar et al. (2007) in both radio and X-ray

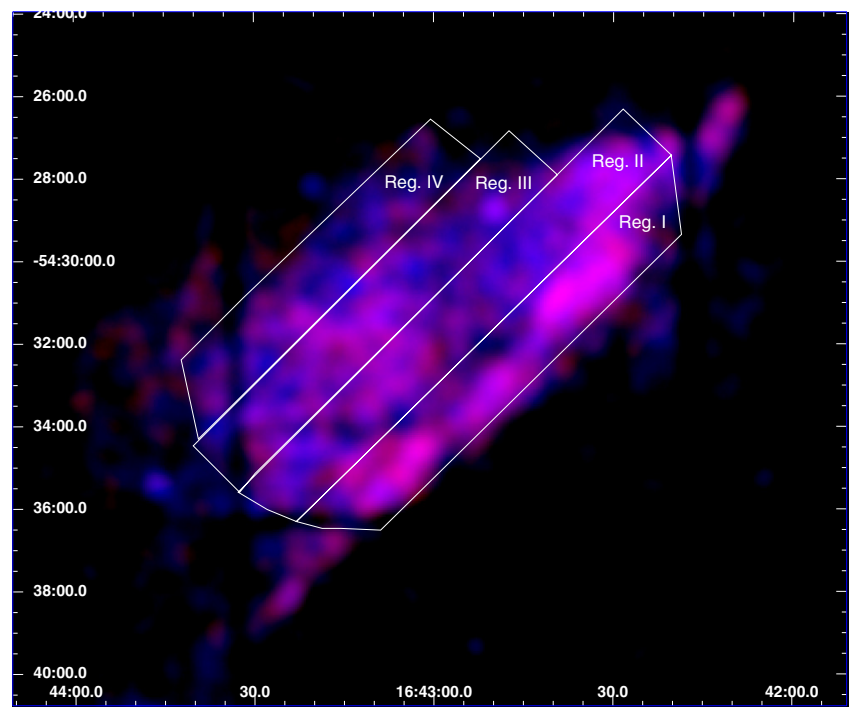

Fig. 1. MOS2 image of the inner section of SNR G332.5-5.6 in soft $\mathrm{X}$-ray energy range. Regions with energies between $0.3-0.6 \mathrm{keV}$ and $0.6-1.0 \mathrm{keV}$ are shown in red and blue, respectively. The regions selected for the spectral analysis are also indicated in white. In this image, north is up and east is to the left.

bands. The central structure is elongated in the NW-SE direction. We observe a plane-like feature, which resembles a wall seen edge-on, toward the west. While diffuse emission is seen extending toward the east.

In the medium and hard X-ray energy range, where no X-ray emission of the SNR is detected, the observations present straylight contamination due to an off-axis source. As the SNR's emission is only appreciable at $E \leq 1.2 \mathrm{keV}$, the spatial and spectral analysis of the X-ray data is not affected by this phenomenon.

\subsection{Mean photon energy map}

To study the physical properties of the X-ray emitting gas, the anisotropy of the total emission, and to get an insight of the thermal structure toward the central part of the SNR, we computed the mean photon energy (MPE) map (Miceli et al. 2005). This MPE map is an image where each computed pixel corresponds to the mean energy of the photons detected by PN CCDs in the 0.5-1.0 keV energy band. Beyond this limit no X-ray emission was detected from the SNR. It provides information about the spatial distribution of the thermal properties of the plasma. Since this characteristic is independent of the spectral model, it cannot be corrected by absorption, and the results are biased toward hard X-rays.

To compute this map, we used the PN event file and created an image with a bin size of $9^{\prime \prime}$, which collects a minimum of 8 counts per pixel everywhere in the remnant. For each pixel, we calculated the mean energy of the photons by means of an IDL script (Bocchino \& Miceli 2004; Miceli et al. 2008) and then we smoothed the map by using a Gaussian kernel value of $3 \sigma$. The smoothed MPE map of the central region of G332.5-5-6 is shown in Fig. 2. According to this map, we are able to confirm that the bulk of the X-ray emission from the SNR has a mean photon energy of $\sim 0.73 \pm 0.05 \mathrm{keV}$, whereas a small part at the center of the shocked structure has a median energy of $0.65 \pm 0.04 \mathrm{keV}$. In a few words, the whole X-ray emission is pretty uniform on the extended emission, with an 


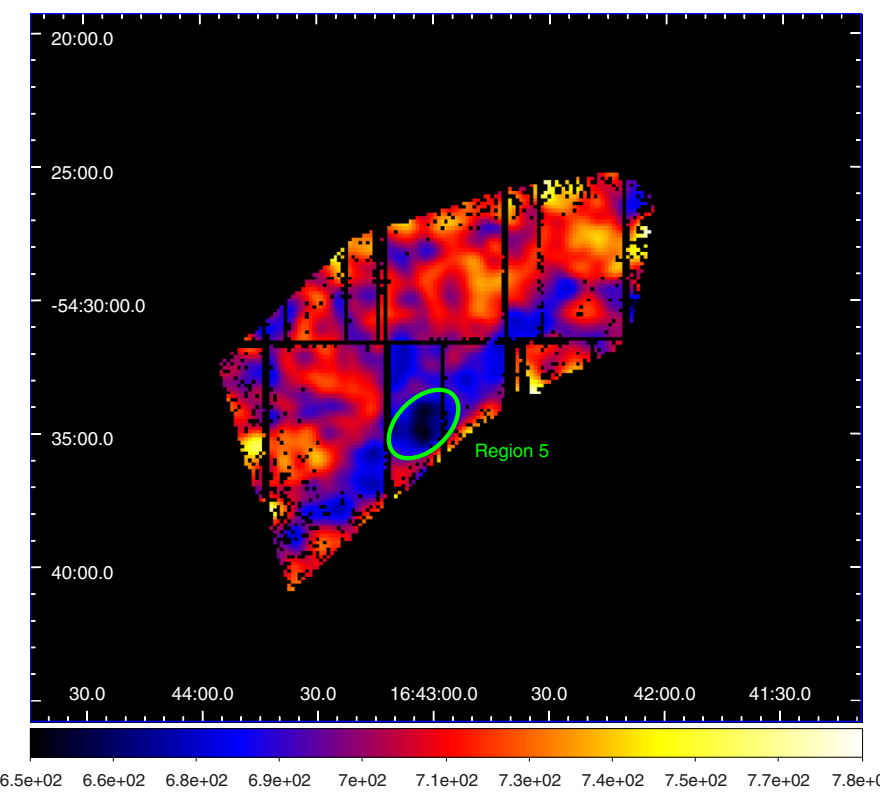

Fig. 2. PN mean photon energy map of the $0.5-1.0 \mathrm{keV}$ emission (bin size $\left.=9^{\prime \prime}\right)$. Pixels with less than 8 counts have been masked out. The color bar has a linear scale and the color-coded energy range is between $0.65 \mathrm{keV}$ and $0.78 \mathrm{keV}$.

$\mathrm{X}$-ray range of emission lower than $0.8 \mathrm{keV}$. This indicates that the post-shocked region at the edge of the SNR had enough time to be uniformly thermalized throughout the interaction with the ISM (see next section).

\subsection{X-ray spectral analysis}

As a result of the high spatial resolution and sensitivity of the $\mathrm{X}$-ray data, we were able to perform an analysis of the observed extended emission. Based on results of our MPE study, first we extracted a single X-ray spectrum of the whole SNR. For this purpose we made use of the XMM-Newton Extended Source Analysis Software (ESAS) tasks (Snowden et al. 2004). We only took spectra from MOS2 and PN cameras into account (see Sect. 3.1). The spectra for background emission were subtracted taking into consideration circular regions in the different CCDs, where no X-ray emission is detected. Ancillary response files (ARFs) and redistribution matrix files (RMFs) were calculated.

Second, to analyze the behavior of the X-ray emission, we performed a spatially resolved spectral study of the central emission of the remnant. In this case, we extracted X-ray spectra of four regions (as indicated in Fig. 1). These regions were chosen to map the change of spectral properties along the SE-NW direction that seems to be the orthogonal direction to the shock front and the dense gas and dust structure observed in the IR band (see Sect. 3.4). In Fig. 3, we show the background subtracted X-ray spectra of the four regions indicated in Fig. 1. The spectra are grouped with a minimum of 16 counts per bin. Error bars were quoted at $90 \%$ and $\chi^{2}$ statistics were used. The spectral analysis was performed using the X-Ray Spectral Fitting Package (XSPEC; Arnaud 1996).

The spectra of the regions were fitted by a VNEI emission model, which is compatible with a thin thermal plasma origin. The model was multiplicatively affected by the TuebingenBoulder ISM absorption model (TBABS; Wilms et al. 2000). We left free abundances of $\mathrm{N}, \mathrm{Ne}, \mathrm{O}$, and $\mathrm{Fe}$. All other

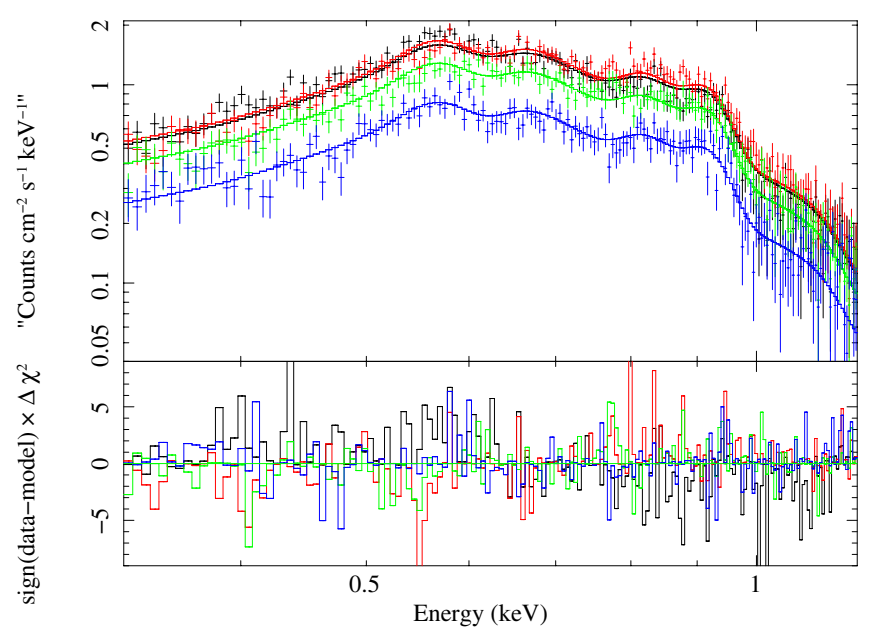

Fig. 3. X-ray spectra from PN data, color refers to regions 1 (black), 2 (red), 3 (green) and 4 (blue). Solid lines indicate the best-fit VNEI model (see Table 1). Lower panels present the $\chi^{2}$ fit residuals.

element abundances were fixed at their solar values. The X-ray parameters of the best fit to the diffuse emission spectra for the different regions are presented in Table 1 .

The spatially resolved spectral analysis shows that the physical conditions of the plasma are clearly homogeneous throughout the central region of the remnant. The mean value of the absorbing column density $N_{\mathrm{H}}$ is about $0.26( \pm 0.02) \times$ $10^{22} \mathrm{~cm}^{-2}$. Similarly, the temperature remains at a mean value of $0.45 \mathrm{keV}$. The uncertainty increase from region 1 to 4 is due to the effect of a lower photon statistic in the X-ray spectra. The values of ionization timescale in all regions are similar, of the order of $\sim 2 \times 10^{10} \mathrm{~s} \mathrm{~cm}^{-3}$. This result is consistent with a nonionization equilibrium state. The relative abundances of the emission are clearly subsolar with a mean of about 0.4 solar value (see Fig. 4). These results are consistent with the analysis performed by Zhu et al. (2015) using Suzaku data. We also found that the absorption corrected flux $F_{\mathrm{X}}$ is large in regions where the X-ray brightness is strong, which is due to intrinsic properties of the SNR and is not related to the absorbing column material located in the line of sight to the remnant. In this case, we would not expect any link between the $N_{\mathrm{H}}$ and the X-ray brightness.

Additional spectra were taken from the region 5, which is within Region 1, as indicated in green in the MPE map (see Fig. 2). These spectra were fitted with a VNEI model, adjusting the abundance parameters to those of Region 1 . The resulting fit showed a lower temperature $k T=0.25 \pm 0.02$, and larger $\tau_{\mathrm{u}}=9.04 \pm 3.0 \times 10^{10} \mathrm{~s} \mathrm{~cm}^{-3}$ than those in Region 1. This is consistent with the shock being slowed down by the interaction with a denser medium. The resulting parameters can be seen in Table 1 in the Region 5 column.

\subsection{Analysis of the cold ISM}

Molecular lines and infrared observations toward SNRs can reveal the presence of molecular/dust material, which can affect the SNR expansion. To study the ISM toward the SNR G332.5-5.6, we look for molecular and IR surveys covering this region of the Galaxy. The only data found with adequate angular resolution to perform this kind of study are from WISE at 3.4, 4.6, 12, and $22 \mu \mathrm{m}$. Therefore, we use these data to find any possible connection with the X-ray emission morphology observed by XMM-Newton. The left pane of Fig. 5 shows 
Table 1. Spectral parameters of the diffuse X-ray emission of the selected regions.

\begin{tabular}{l|llllll}
\hline \hline Model and parameters & Total & Region 1 & Region 2 & Region 3 & Region 4 & Region 5 \\
\hline TBABS*VNEI & & & & & & \\
$N_{\mathrm{H}}\left[10^{22} \mathrm{~cm}^{-2}\right]$ & $0.26 \pm 0.02$ & $0.28 \pm 0.02$ & $0.29 \pm 0.03$ & $0.25 \pm 0.03$ & $0.25 \pm 0.02$ & $0.28^{\dagger}$ \\
$k T[\mathrm{keV}]$ & $0.45 \pm 0.07$ & $0.42 \pm 0.07$ & $0.41 \pm 0.05$ & $0.45 \pm 0.07$ & $0.54 \pm 0.14$ & $0.25 \pm 0.01$ \\
$N\left[N_{\odot}\right]$ & $0.57 \pm 0.24$ & $0.59 \pm 0.21$ & $0.40 \pm 0.18$ & $0.43 \pm 0.21$ & $0.24 \pm 0.14$ & $0.59^{\dagger}$ \\
$\mathrm{O}\left[\mathrm{O}_{\odot}\right]$ & $0.35 \pm 0.06$ & $0.34 \pm 0.04$ & $0.28 \pm 0.04$ & $0.32 \pm 0.06$ & $0.24 \pm 0.03$ & $0.34^{\dagger}$ \\
$\mathrm{Ne}\left[\mathrm{Ne}_{\odot}\right]$ & $0.48 \pm 0.11$ & $0.40 \pm 0.06$ & $0.35 \pm 0.05$ & $0.46 \pm 0.08$ & $0.35 \pm 0.05$ & $0.40^{\dagger}$ \\
$\mathrm{Fe}\left[\mathrm{Fe}_{\odot}\right]$ & $0.40 \pm 0.09$ & $0.37 \pm 0.06$ & $0.31 \pm 0.04$ & $0.36 \pm 0.06$ & $0.31 \pm 0.06$ & $0.37^{\dagger}$ \\
$\tau_{u}\left[10^{10} \mathrm{~s} \mathrm{~cm}^{-3}\right]$ & $2.03 \pm 0.48$ & $1.70 \pm 0.73$ & $2.89 \pm 0.77$ & $2.53 \pm 0.72$ & $1.22 \pm 0.50$ & $9.04 \pm 3.04$ \\
Norm $\left[10^{-4}\right]$ & $55.84 \pm 18.1$ & $24.7 \pm 10.3$ & $28.8 \pm 10.6$ & $12.1 \pm 5.11$ & $9.36 \pm 3.84$ & $6.03 \pm 1.07$ \\
\hline$\chi_{v}^{2} /$ d.o.f. & $1.29 / 689$ & $1.10 / 188$ & $1.00 / 189$ & $1.01 / 191$ & $0.99 / 187$ & $1.15 / 226$ \\
\hline Flux(0.3-1.0 keV) $\left[10^{-12}\right]$ & 30.46 & 11.35 & 9.46 & 5.89 & 5.31 & 0.13 \\
\hline
\end{tabular}

Notes. Normalization is defined as $10^{-14} / 4 \pi D^{2} \times \int n_{\mathrm{H}} n_{\mathrm{e}} \mathrm{d} V$, where $D$ is distance in [cm], $n_{\mathrm{H}}$ is the hydrogen density [ $\left.\mathrm{cm}^{-3}\right], n_{\mathrm{e}}$ is the electron density $\left[\mathrm{cm}^{-3}\right]$, and $V$ is the volume $\left[\mathrm{cm}^{3}\right]$. Fluxes are absorption-corrected and error values are in the $90 \%$ confidence interval for every single parameter, and they are given in units of $10^{-13} \mathrm{erg} \mathrm{cm}^{-2} \mathrm{~s}^{-1}$. Abundances are given relative to the solar values of Anders \& Grevese (1989). (†) These parameters were fixed to the fit paramenters from Region 1.
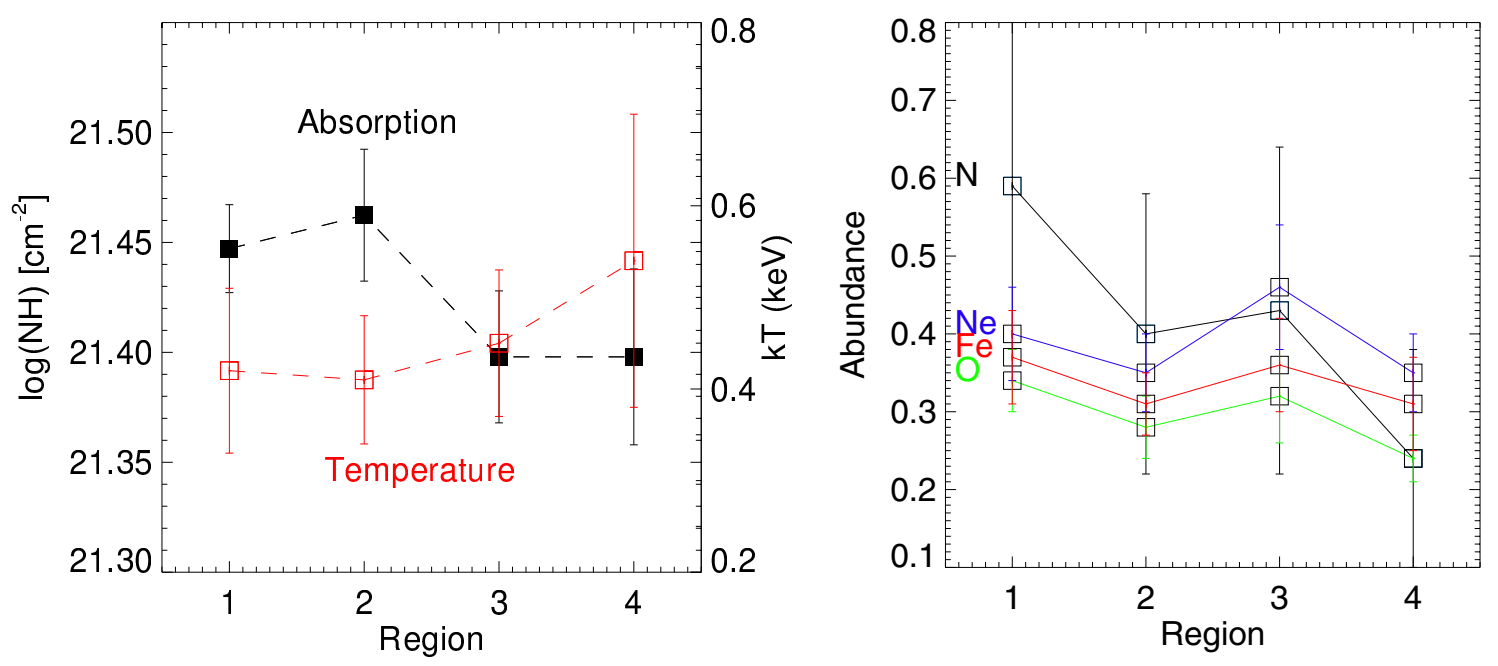

Fig. 4. X-ray parameters obtained from the spectral analysis as a function of the distance from the plane-like region increasing toward the northwest direction. Left: the absorption-temperature behavior seems to be the same inside the error, with a mean of $2.6 \times 10^{21} \mathrm{~cm}^{-2}$ and $0.45 \mathrm{keV}$ for total of emission from the SNR. Right: abundance of N, Ne, Fe, and $\mathrm{O}$ are clearly subsolar with a median about $0.4 \pm 0.07$.

a composition with WISE bands at 12 and $24 \mu \mathrm{m}$ in green and red, and X-ray emission in blue. In contrast, the right panel of Fig. 5 shows IR emission taken by WISE in the bands 4.6, 12, and $24 \mu \mathrm{m}$ in blue, green, and red, respectively, with the soft $\mathrm{X}$-ray contours superimposed in yellow. As can be seen from the first image, the X-ray emission (blue) does not display a clear counterpart in the IR bands, which could be originated in synchrotron emission, shock-heated dust, atomic fine-structure lines, or molecular lines (see, e.g., Reach et al. 2006). However, it is important to note that the plane-like region observed on the western part of the X-ray emission (also observed in the radio bands) coincides with the edge of a bright structure detected in mid-IR. This sharp edge seen in the X-ray emission can be interpreted as the shock interacting with a denser medium (bright in infrared), which is hampering the expansion of the remnant. This plane-like structure can be the result of the expansion of the shock in a medium with different possible geometries (i.e., a cylindrical cavity or plain layer), which encounters a denser material. The fact that we only observe the interacting part in $\mathrm{X}$-ray may be due to reflected shock produced by the impact with the cloud, which makes the interacting region brightest since it has been shocked and heated twice. The soft X-ray emission, represented in blue in on the left side of Fig. 5, seems to coexist with this IR emission, as seen toward the west. This suggests that the molecular cloud interacting with the SNR is coexisting with the remnant or is in front of it. Assuming that the mid-IR emission arises from dust and polycyclic aromatic hydrocarbon (PAH) molecules, we suggest that the expansion occurred in a less dense medium during the early evolutionary phase and then the forward shock encountered a more dense medium of gas and dust, as observed at IR wavelengths. To complement this, it would be necessary to observe this region in some molecular lines, such as ${ }^{12} \mathrm{CO},{ }^{13} \mathrm{CO}$ and $\mathrm{C}^{18} \mathrm{O} J=1-0,2-1$, and $3-2$, to search for the presence of molecular gas, and in case of positive detection, perform a study of its physical conditions.

Finally, to find if there exists some contribution of a compact central source to the X-ray emission observed on the central part of the SNR, we ran a source detection task EDETECT-CHAIN using SAS for the whole X-ray energy range. As a result, we found six point-like X-ray sources, which also present IR emission in WISE data. After a spectral X-ray analysis of these sources we found that none display typical central compact object (CCO) characteristics. In Table 2, the Equatorial coordinates of the sources, which have an IR counterpart, are listed together 
Table 2. X-ray sources with IR counterpart.

\begin{tabular}{ccc}
\hline \hline$\alpha$ & $\delta$ & Probable spectral type \\
\hline 164135.06 & $-543017.29,36^{\prime \prime}$ & YSO \\
164253.04 & $-543919.44,36^{\prime \prime}$ & YSO \\
164250.40 & $-542845.12,36^{\prime \prime}$ & O-type \\
164312.24 & $-543333.84,36^{\prime \prime}$ & $\mathrm{B}(0-5)$ \\
164346.32 & $-543525.44,36^{\prime \prime}$ & $\mathrm{B}(0-5)$ \\
164414.88 & $-542909.60,36^{\prime \prime}$ & O-type \\
\hline
\end{tabular}

Notes. List of X-ray sources with IR counterpart. The first two columns have the equatorial coordinates of the sources and the third the spectral type.

with the spectral types. The latter are the result of a preliminary photometric analysis based on WISE and 2MASS data. The YSO candidates were determined from the criteria presented by Koenig et al. (2012), based on the magnitudes measured in WISE bands. The OB-type star candidates were found from a typical color-magnitude diagram using 2MASS bands.

We calculated the threshold counts expected at the PN camera from a putative $\mathrm{CCO}$ immersed in the SNR. Considering that the lowest luminosity for a CCO is $\sim 10^{32} \mathrm{erg} \mathrm{s}^{-1}$ (Halpern et al. 2010), and assuming a distance of $3.4 \mathrm{kpc}$ to the SNR, we obtain a minimum flux for a CCO of $\sim 7.6 \times 10^{-14} \mathrm{erg} \mathrm{cm}^{2} \mathrm{~s}^{-1}$. Using WebPIMMS tool ${ }^{1}$, we can calculate a count rate for this flux for XMM-Newton. Assuming an absorption column density of $n_{\mathrm{H}} \sim 0.2 \times 10^{22}$ and a blackbody model source with $0.5 \mathrm{keV}$ temperature, we obtain a count rate for $\mathrm{PN}$ camera (with medium filter) of $4.597 \times 10^{-2}$ cts s$^{-1}$ (counts per second). Taking into consideration that we have a $27.1 \mathrm{ks}$ GTI for PN camera with medium filter, we expect at least $\sim 1200$ counts for a CCO. In conclusion, as we did not have this kind of detection we can assure with a high level of confidence, that there is no CCO in this region.

We found evidence of dense material associated with IR emission on the west part of the central region of the SNR. In this case, the passage of the shock front could have interacted with interstellar material generating gamma-ray emission. To search for evidence of this possibility, Fermi-LAT data were used to search (unsuccessfully) for gamma-ray emission (see Appendix A for details).

\section{Discussion}

SNR G332.5-5.6 has been previously classified as a thermal composite or mixed morphology SNR by Reynoso et al. (2007). These kind of remnants are characterized as having radio shells and central X-ray emission, which can peak or be amorphous in reference to the center of the source. This X-ray emission is from thermal origin and product of swept-up interstellar material instead of ejecta. This can be seen by the appearance of lines in the spectra. Parameters like temperature, show uniformity along the remnant, density presents higher values inside the remnant than in the exterior, and metal abundances take values similar to solar abundances. Another important characteristic is that these kind of remnants are usually seen interacting with molecular or HI clouds (Rho et al. 1998).

In the case of G332.5-5.6 the central X-ray emission has a clear correlation with the central radio emission, however, radio emission is trident-like, not shell-like. Temperature remains almost constant in all regions. A similar behavior can be observed

\footnotetext{
1 https://heasarc.gsfc.nasa.gov/cgi-bin/Tools/

w3pimms/w3pimms.pl
}

for density parameter, which does not have appreciable changes. Regarding metal abundances, most of metal abundances are solar-like and although $\mathrm{N}, \mathrm{O}, \mathrm{Ne}, \mathrm{Fe}$, present subsolar abundances, they remain near solar values. Infrared data confirm the presence of atomic and molecular/dust material. With this information in mind, we suggest that G332.5-5.6 is an unusual MM subclass category of SNR

The results of the multiwavelength data analysis presented above can be summarized as follows:

- The morphology of the extended soft X-ray emission detected by XMM-Newton from the central part of the SNR, is thermal, under nonequilibrium state, and matches very well the emission observed at different radio frequencies. Given the low abundances we can associate the X-ray emitting plasma with shocked ISM and not ejecta.

- The most striking feature is observed on the western part of the emission, where a plane-like region or interface between two different medium seems to exist.

- The region to the right part of this interface shows the presence of enhanced IR emission merged with very weak X-ray emission.

- No point sources with CCOs characteristics were detected in the central region of the SNR.

These results seem to support a scenario where the distorted morphology of the remnant is caused by the expansion of the shock front, first through a low density ISM and then encountering a region with higher density of dust located on the west part of the remnant.

As the SNR evolves, it compresses and heats up the surrounding medium to $\mathrm{X}$-ray emitting temperatures. When the shock front encounters a high density region, its velocity diminishes and the temperature of the shocked plasma can be considerably lower. In this case, the expansion of the remnant can be significantly impeded, leading to the observed morphology. In some cases, the apparent morphologies of SNRs can depend upon the density and distribution of foreground material. For example, in the case of presence of large values of $N_{\mathrm{H}}$ associated with large distances through the ISM, the observed X-ray emission is faint, or the presence of molecular clouds along the line of sight to an SNR produces excess absorption.

Since the X-ray emission does not originate from the whole SNR, but only from a small part of it (in fact, the radio extension of the SNR is much larger than its X-ray radius), the XMM-Newton observations can only be used to obtain some physical properties of the plasma responsible for the X-ray emission. Therefore assuming that the emitting plasma fills a box with an extension along the line of sight comparable with its extension in the plane of the sky (i.e., 5-6 arcmin), we obtain a volume $V$ of $3.5 \times 10^{57} \mathrm{~cm}^{3}$ at a mean distance of $3.4 \mathrm{kpc}$ (which is in agreement with the recent studies performed by Zhu et al. 2015). Using the emission measure (EM) determined from the spectral fitting, we estimated the electron density of the plasma $n_{\mathrm{e}}=\sqrt{E M / V}$ to be $0.4 \mathrm{~cm}^{-3}$. In this case, the density of the nucleons was simply assumed to be the same as that of electrons.

The age, for the X-ray emitting plasma, $t_{\text {plasma }}$ was then determined using the upper limit ionization timescale, $\tau_{\mathrm{ul}}$, by $t=\tau_{\mathrm{ul}} / n_{\mathrm{e}}$. As a result, the elapsed time after the plasma was heated is $\sim 1.4 \times 10^{3} \mathrm{yr}$. The total mass of the X-ray emitting plasma $M_{\text {total }}$ can be estimated by

$M_{\text {total }}=n_{\mathrm{e}} V m_{\mathrm{H}} \sim 1.4 M_{\odot}$,

where $m_{\mathrm{H}}$ is the mass of a hydrogen atom. 

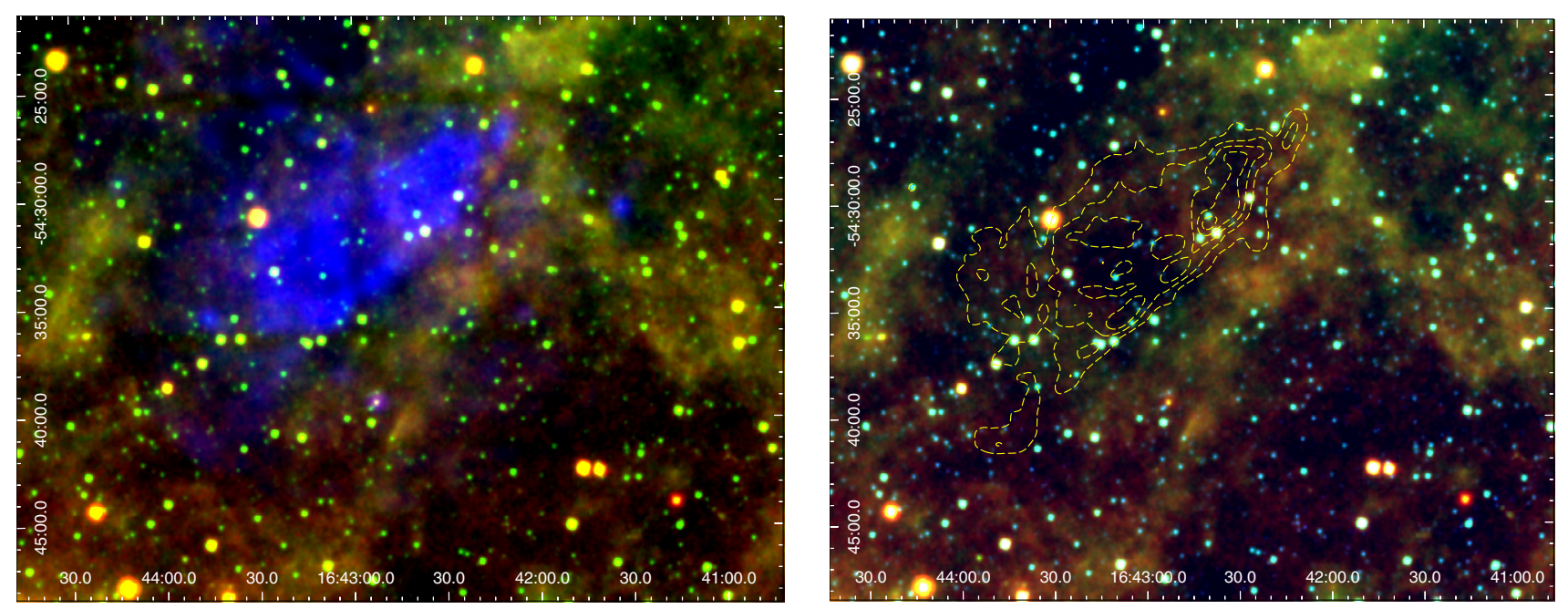

Fig. 5. Left panel: infrared observations of WISE bands at 12 and $24 \mu \mathrm{m}$ are shown in green, and red, respectively, with the soft X-ray emission superimposed in blue. Right panel: infrared observations of WISE bands at 4.6, 12 and $24 \mu \mathrm{m}$ are shown in blue, green, and red, respectively. The soft X-ray contours are superimposed in yellow.

\section{Conclusions}

In this work, we present a detailed analysis of the X-ray observations obtained with the XMM-Newton telescope of the central region of the SNR G332.5-5.6, along with infrared data available in the literature. The results show that the X-ray emission peaks in the soft energy range $(0.3-1.0 \mathrm{keV})$, and confirms that the X-ray morphology has a good correlation with radio emission detected at different frequencies on the central part of the SNR.

From the spatially spectral X-ray analysis, we found that the spectra is thermal and can be well fitted by a VNEI model with a subsolar abundance of $\mathrm{N}, \mathrm{O}, \mathrm{Ne}$, and Fe. The IR observations suggest that the distorted morphology of the central radio/X-ray emission of the remnant could be the result of the expansion of the shock front, first through a thin ISM and then encounter a region with higher density of dust located on the west of the remnant. All this evidence, suggests that the morphology seen is strongly coupled to the local physical conditions of the ISM.

While the paper by Zhu et al. (2015) provides a global description of the physical properties of the X-ray emitting plasma in (part of) the central region of the remnant, our XMM-Newton data allowed us to perform a spatially resolved spectral analysis, thus revealing the presence of a peculiar region (region 5) characterized by soft X-ray emission, indicative of dense plasma. Further X-ray observations of the central region of this SNR performed by Chandra, at high spatial resolution, are necessary to better understand the nature and morphology of the central part of the SNR.

Acknowledgements. We thank the anonymous referee for her/his insightful comments and constructive suggestions that led to an improved manuscript. A.E.S and F.G. are fellows of CONICET, J.A.C. and J.F.A.-C. are researchers of CONICET. J.A.C. was supported for different aspects of this work by grants AYA2013-47447-C3-3-P from the Spanish Government, Consejería de Economía, Innovación y Ciencia de Junta de Andalucía as part of research group FQM-1343, excellence fund FQM-5418, as well as FEDER funds. J.F.A.C. was supported by grant PIP 2014-0285 (CONICET). S.P. is supported by CONICET, ANPCyT, and UBA (UBACyT) grants.

\section{Appendix A: Fermi data analysis}

To search any possible gamma-ray emission observed by Fermi telescope, we used $\gamma$-ray data from Fermi Large Area Telescope (LAT) to detect any possible gamma-ray emission in the region centered at the extended X-ray emission $\left(\alpha=16^{\mathrm{h}} 42^{\mathrm{m}} 55^{\mathrm{s}} .0\right.$, $\left.\delta_{\mathrm{J} 2000.0}=-54^{\circ} 31^{\prime} 00^{\prime} \cdot 0\right)$ within a circle of $4^{\circ}$ of radius (ROI; region of interest).

The data analysis was performed using the Fermi Science Tools package (v9r32p5) available from the Fermi Science Support Center (FSSC). The data was obtained from the reprocessed Fermi Pass 7 database and the instrumental response function used was the P7REP SOURCE V15 version. We analyzed six years of Fermi data, from August 2008 to August 2014 (2008-08-04T15:43:36 to 2014-08-28T08:34:21, UTC). To prevent the contamination by the Earth's albedo, the events with zenith angle greater than $100^{\circ}$ or rocking angle greater than $52^{\circ}$ were filtered. Unbinned likelihood analysis was performed using the gtlike function. To model the background source contributions, we included the entire two-year Fermi gamma-ray LAT (2FGL) catalog of point sources (Nolan et al. 2012) associated with the extended source templates within $4^{\circ}$ from the ROI center.

The galactic diffuse background (gll_iem_05.fits) and the isotropic diffuse background (iso_sourcev05.txt) were also included in the modeling. All of these background modeling resources are available from the FSSC. Using the full energy range extracted, $100 \mathrm{MeV}$ to $300 \mathrm{GeV}$, we modeled the differential gamma-ray flux expected from a point source located at the center of our ROI using a simple power law

$$
\frac{\mathrm{d} N}{\mathrm{~d} E}=N_{0}\left(\frac{E}{E_{0}}\right)^{-\alpha},
$$

where $N_{0}$ is the normalization factor, $E_{0}$ is the energy scale, and $\alpha$ the spectral index.

There is no detection by the Fermi-LAT in the chosen energy band at the location of the extended central X-ray source, therefore, only upper limits for the gamma-ray emission could be determined from this data. The value found for this upper limit is of $4.7 \times 10^{-7} \mathrm{ph} \mathrm{cm}^{-2} \mathrm{~s}^{-1}$ between $100.0 \mathrm{MeV}$ and $30 \mathrm{GeV}$. 
A. E. Suárez et al.: XMM-Newton observations of G332.5-5.6

\section{References}

Anders, E., \& Grevesse, N. 1989, Geochim. Cosmochim. Acta, 53, 197 Arnaud, K. A. 1996, Astronomical Data Analysis Software and Systems V, 101, 17

Bocchino, F., Miceli, M., \& Maggio, A. 2004, Adv. Space Res., 33, 381 Bock, D. C.-J., Large, M. I., \& Sadler, E. M. 1999, AJ, 117, 1578

Griffith, M. R., \& Wright, A. E. 1993, AJ, 105, 1666

Halpern, J. P., \& Gotthelf, E. V. 2010, ApJ, 709, 436

Koenig, X. P., Leisawitz, D. T., Benford, D. J., et al. 2012, ApJ, 744, 130

Miceli, M., Bocchino, F., Maggio, A., \& Reale, F. 2005, A\&A, 442, 513

Miceli, M., Decourchelle, A., Ballet, J., et al. 2008, Adv. Space Res., 41, 390

McClure-Griffiths, N. M., Pisano, D. J., Calabretta, M. R., et al. 2009, ApJS, 181,398

Nolan, P. L., Abdo, A. A., Ackermann, M., et al. 2012, ApJS, 199, 31
Parker, Q. A., Phillipps, S., Pierce, M. J., et al. 2005, MNRAS, 362, 689 Reach, W. T., Rho, J., Tappe, A., et al. 2006, AJ, 131, 1479

Reynoso, E. M., \& Green, A. J. 2007, MNRAS, V375, 92

Rho, J., \& Petre, R. 1998, ApJ, 503, L167

Skrutskie, M. F., Cutri, R. M., Stiening, R., et al. 2006, AJ, 131, 1163

Snowden, S., Valencic, L., Perry, B., Arida, M., \& Kuntz, K. D. 2004, The $X M M$-Newton ABC Guide: an introduction to XMM-Newton Data Analysis, with contributions by: I. Harrus, S. Immler, R. Shafer, R. Smith, \& M. Still, 97 Stupar, M., Parker, Q. A., Filipović, M. D., et al. 2007, MNRAS, V381, 377

Strüder, L., Briel, U., Dennerl, K., et al. 2001, A\&A, 365, L18

Turner, M. J. L., Abbey, A., Arnaud, M., et al. 2001, A\&A, 365, L27

Whiteoak, J. B. Z., \& Green, A. J. 1996, A\&AS, 118, 329

Wilms, J., Allen, A., \& McCray, R. 2000, ApJ, 542, 914

Wright, E. L., Eisenhardt, P. R. M., Mainzer, A. K., et al. 2010, AJ, 140, 1868

Zhu, H., Tian, W. W., \& Wu, D. 2015, MNRAS, 452, 3470 